\title{
Comparing Men who have Sex with Men and Transgender Women who Use Grindr, Other Similar Social and Sexual Networking Apps, or no Social and Sexual Networking Apps: Implications for Recruitment and Health Promotion
}

Christina J Sun ${ }^{1 *}$, Erin L Sutfin², Laura H Bachmann ${ }^{3}$, Jason Stowers ${ }^{4}$ and Scott D Rhodes ${ }^{2}$

${ }^{1}$ School of Public Health, Oregon Health \& Science University-Portland State University, Portland, OR, USA

${ }^{2}$ Division of Public Health Sciences, Department of Social Sciences and Health Policy, Wake Forest University School of Medicine, Winston-Salem, NC, USA

${ }^{3}$ Infectious Diseases Section, Wake Forest University Health Sciences, Winston-Salem, NC, USA

${ }^{4}$ Triad Health Project, Greensboro, NC, USA

\begin{abstract}
Objective: Researchers and public health professionals have increased their attention to GPS-based social and sexual networking applications (apps) tailored to gay, bisexual, and other men who have sex with men (MSM) and transgender women. These populations continue to be disproportionately affected by HIV in the United States; therefore, these apps, in particular Grindr, have become an important sampling venue for the recruitment of HIV-related research participants. As such, it is essential to identify differences among app users to avoid potential sampling bias. This paper seeks to identify differences in MSM and transgender women who use Grindr and those who use other similar apps.
\end{abstract}

Methods: A community-based participatory research (CBPR) approach was used to recruit participants online who then completed a 25-item anonymous survey. Five domains were assessed: sociodemographics, HIV testing, sexual risk, substance abuse, and use of GPS-based social and sexual networking apps.

Results: 457 participants completed surveys. There were significant differences in the sociodemographic characteristics by app use, including age, race/ethnicity, sexual orientation, and outness. After adjusting for the sociodemographic characteristics associated with app use, there were significant differences in HIV risk and substance use between the groups.

Conclusion: This paper is the first to report on findings that compare MSM and transgender women who report using Grindr to MSM and transgender women who report using other similar apps. GPS-based social and sexual networking apps may offer a valuable recruitment tool for future HIV research seeking to recruit populations at increased risk for HIV or those living with HIV for therapeutic trials. Because of the differences identified across users of different apps, these findings suggest that if researchers recruited participants from just one app, they could end up with a sample quite different than if they had recruited MSM and transgender women from other apps.

Keywords: HIV; Men who have sex with men; MSM; Transgender women; Mobile applications; Apps; Community-based participatory research; Sampling

\section{Introduction}

Recently, researchers and public health professionals have increased their attention to GPS-based applications ("apps") and other social media platforms that are tailored to gay, bisexual, and other men who have sex with men (MSM) and transgender women, groups that continue to be most disproportionately affected by HIV in the United States (US) [1-3]. Some social media platforms, such as GPSbased mobile apps, incorporate global positioning system technology to facilitate in-person networking. MSM and transgender women reported using apps for a variety of reasons, including friendship and social support [4]. Significant proportions of MSM also report using these apps for sexual networking and recent estimates suggest that oneto two-thirds of MSM use these apps to meet sexual partners [5-7]. Several studies found app users to be more likely to report more sexual partners and condomless anal intercourse and to be at increased risk for sexually transmitted infections (STIs), including HIV [6,8-11]. Sexual health promotion, such as to increase awareness of syphilis and syphilis testing [12], distribute HIV self-test kits [13], and increase linkage to HIV testing and treatment services at a local health department [14], have successfully occurred on these apps. Most of these efforts have occurred on one app in particular, specifically, Grindr, which was the first GPS-based social and sexual networking app of its kind. Additionally, most of our understanding of MSM who use GPS-based social and sexual networking apps has been based on samples recruited from Grindr [4,7,11,15-19], despite since its launch in 2009, many similar apps have been developed and some of these apps have also been tailored for various sub-communities and interests [20].

When the Internet emerged as an important way for people to connect, researchers also recruited samples via the Internet. At that time, questions were raised about the potential differences that may exist between Internet site users. Researchers have identified significant

*Corresponding author: Christina J Sun, PhD, MS, OHSU-PSU School of Public Health, Suite 450H, 506 SW Mill St., Portland, OR 97201, USA, Tel: 503-725-3616; Fax: 503-725-5100; E-mail: christina.sun@pdx.edu

Received February 07, 2018; Accepted February 15, 2018; Published February 22, 2018

Citation: Sun CJ, Sutfin EL, Bachmann LH, Stowers J, Rhodes SD (2018) Comparing Men who have Sex with Men and Transgender Women who Use Grindr, Other Similar Social and Sexual Networking Apps, or no Social and Sexual Networking Apps: Implications for Recruitment and Health Promotion. J AIDS Clin Res 9: 757. doi: 10.4172/2155-6113.1000757

Copyright: ( 2018 Sun CJ, et al. This is an open-access article distributed unde the terms of the Creative Commons Attribution License, which permits unrestricted use, distribution, and reproduction in any medium, provided the original author and source are credited. 
Citation: Sun CJ, Sutfin EL, Bachmann LH, Stowers J, Rhodes SD (2018) Comparing Men who have Sex with Men and Transgender Women who Use Grindr, Other Similar Social and Sexual Networking Apps, or no Social and Sexual Networking Apps: Implications for Recruitment and Health Promotion. J AIDS Clin Res 9: 757. doi: 10.4172/2155-6113.1000757

Page 2 of 6

differences in participant characteristics depending on how and from which online venues they were recruited [20,21]. Akin to the Internet, GPS-based social and sexual networking apps have become popular social media platforms, thus sampling venues, of this part of the 21st century. As such, there is an increasing need to understand apps as sampling venues and currently, there has not been systematic exploration of differences among various app users. Considering the potential differences between MSM and transgender women who use Grindr and those who instead use similar apps, we sought to examine if recruiting MSM and transgender women exclusively from Grindr unintentionally contributes to sampling bias. This analysis sought to address this gap by comparing the sociodemographic characteristics, HIV testing, sexual risk, and substance use of MSM and transgender women who do and do not use Grindr and other similar apps.

\section{Methodology}

\section{Community-based participatory research}

A community-based participatory research (CBPR) partnership, including community members, organization representatives, and academic researchers, conducted this study. The partners have worked together for over 15 years to design studies to explore and promote sexual and reproductive health and reduce HIV risk among a variety of health disparity populations [22]. In a recent study that we conducted together to test the acceptability and feasibility of providing HIV and STI testing referrals via four GPS-based social and sexual networking apps, the health educator observed sociodemographics differences in the profiles of the users across the apps [23].

\section{Study design}

Using a previously validated recruitment strategy [24-27], we recruited participants from four metropolitan areas across North Carolina (i.e., Asheville, Charlotte, Greensboro, and Greenville) to complete a 25-item anonymous internet survey. Participants were recruited through four social media platforms designed to facilitate social and sexual networking among MSM and transgender women: Craigslist, Adam4Adam, Black Gay Chat, and Gay.com. Craigslist offers different websites for different cities and their surrounding areas where users can post classified advertisements (such as for jobs, items for sale, and housing) and a personals sections. We posted an invitation to participate in the survey in the "casual encounters $\mathrm{m} 4 \mathrm{~m}$ [men for men]" and "men seeking men" personals subcategories. Interested users then responded by emailing the person who posted the ad or personals. Adam4Adam, Black Gay Chat, and Gay.com allow users to create profiles including their pictures and a description of themselves. Within these platforms, users can set filters to find other users within a certain geographic region. Users can then select and read profiles of other users and if interested can initiate a chat to engage in a one-on-one conversation. We used standardized language across all four websites that stated we were recruiting participants to complete a short survey and requested an interested user email us (in the case of Craigslist) or initiate a chat (in the case of Adam4Adam, Black Gay Chat, and Gay. com) if they were interested to learn more about the study. We did not target or approach users to complete our survey.

Participants were compensated $\$ 10$, which they could obtain through PayPal, donate to a nonprofit of their choice, or forego. Wake Forest School of Medicine Institutional Review Board approved all study procedures (IRB00014308).

\section{Measures}

Five different domains were assessed through the survey: sociodemographics, HIV testing, sexual risk, substance use, and use of GPS-based social and sexual networking apps. All items had been previously validated and used in research conducted by the CBPR partnership [28-31].

Sociodemographics: Participants reported their age, race/ ethnicity, county of residence, gender, sexual orientation, and level of "outness." The sociodemographic measures and response options reflect language used on these sites. In the analyses, the race/ethnicity options (American Indian/Alaska Native, Asian, Black/African American, Hispanic/Latino, Native Hawaiian or other Pacific Islander, White, and Other) were dichotomized as either White or Non-White. Participants described their sexual orientation as bisexual, gay or homosexual, heterosexual or straight, or other. Participants designated their outness by selecting one option that best reflected how "out" they were about their sexual behavior and/or orientation. Options were: "not at all," "not out at work," "to some people," "to all but family," or "totally." No participants chose "not out at work;" therefore, we made the response options of this measure range on a 4-point scale from 1 representing "not at all" to 4 representing "totally." This item reflected how users of these social and sexual networking sites are asked to self-identity.

HIV testing: Participants reported whether they had ever been tested for HIV in their lifetime and in the past 12 months. Participants reported the result of the test with the response options: "negative", "positive", "indeterminate", and "did not receive results." Participants also reported the location of their last HIV test. Location was classified as a traditional venue (e.g., doctor's office) or non-traditional venue (e.g., bar/club and church). HIV testing self-efficacy was measured on a 4-point scale and participants reported how sure they were that they could get tested for HIV, from 1 "not at all sure" to 4 "very sure."

Sexual risk: We used four questions to measure sexual activity and condom use with men and women. Participants reported whether they had oral, vaginal, and/or anal sex with a woman in the past 12 months (yes or no) and whether they had oral and/or anal sex with a man in the past 12 months (yes or no). Condom use during vaginal or anal sex with women in the past 12 months and condom use during anal sex with men during the past 12 months was measured on a 5-point scale, "never," "rarely," "sometimes," "usually," and "always." The selection of "never," "rarely," "sometimes," and "usually," was classified as inconsistent condom use.

Substance use: Alcohol use was measured by asking participants, "In a typical week, how many days do you get drunk?" [30]. Participants also reported which of the following substances they had used in the past 12 months: tobacco (cigarette or cigar), marijuana, cocaine, crack, "poppers" or rush (alkyl nitrites), crystal meth, ecstasy, GHB, and medication used for sexual enhancement (i.e., Viagra ${ }^{\star}$, Levitra ${ }^{\star}$, or Cialis $^{\circ}$ ). Additionally, participants had the option of listing any other drugs they had used in the past 12 months. Recreational prescription drug use was assessed by asking participants if they had ever used prescription drugs without a prescription or for reasons other than the prescribed use.

App use: Participants indicated all of the apps they had used in the past 30 days from a list of 11 GPS-based social and sexual networking apps (i.e., A4A/Radar, BoyAhoy, GayConnect, Grindr, Growlr, GuySpy, Hornet, iDate, Jack'd, Mister, Scruff). If "Other" was selected they were asked to specify the app. 
Citation: Sun CJ, Sutfin EL, Bachmann LH, Stowers J, Rhodes SD (2018) Comparing Men who have Sex with Men and Transgender Women who Use Grindr, Other Similar Social and Sexual Networking Apps, or no Social and Sexual Networking Apps: Implications for Recruitment and Health Promotion. J AIDS Clin Res 9: 757. doi: 10.4172/2155-6113.1000757

Page 3 of 6

\section{Analysis plan}

First, we identified three groups of MSM and transgender women based on their reported app use in the past 30 days. If a participant did not report using a GPS-based social and sexual networking app designed for MSM and transgender women in the past 30 days, the participant was classified as a "non-app user." Participants who reported using Grindr (regardless of other use of other apps) in the past 30 days were classified as a "Grindr user." Participants who did not report using Grindr in the past 30 days, but reported using at least one other GPS-based social and sexual networking apps designed for MSM were classified as a "non-Grindr app user."

We performed descriptive analyses to examine overall prevalence and group prevalence of app use. We compared the sociodemographic characteristics, HIV testing experiences, sexual risk, and substance use of participants by app use (non-app user, Grindr user, and non-Grindr app user) with chi-square tests and Fisher's exact tests for categorical variables and analysis of variance (ANOVA) for continuous variables. Variables that were significantly different in the bivariable analysis $(p<0.10)$ were entered into a multivariable logistic regression model to determine the associations between app use and HIV risk and substance use [32]. All analyses were performed in SPSS 22 (Armonk, NY).

\section{Results}

\section{Description of participants}

Table 1 presents the sociodemographic characteristics of the sample and differences by app use. On average, participants $(\mathrm{n}=457)$ were 41.1 years old $(S D=12.7$, range $=18-74)$. The majority of the sample was White $(82.3 \%)$ and identified as male (98.7\%). Over half the sample identified as bisexual (40.7\%), or heterosexual/other (13.6\%).

Table 1 also presents the HIV risk and substance use behavior for the total sample and by app use. Over three-quarters (78.3\%) of participants had ever been tested for HIV and $45.1 \%$ had been tested in the past 12 months. About two-thirds (60.4\%) of participants reported their last HIV test occurred at a non-traditional testing location. Slightly less than half (46.8\%) of participants reported having sex with a woman in the past 12 months. No participants reported using marijuana, crack, crystal meth, or GHB in the past 12 months. Over one-third (38.7\%) reported tobacco (cigarette or cigar) use and $16.4 \%$ reported using poppers in the past 12 months. About one-fifth (19.0\%) of participants reported using medication for sexual enhancement and $9.6 \%$ reported using prescription medication recreationally.

\begin{tabular}{|c|c|c|c|c|c|}
\hline & Sample $(n=457)$ & Non-app user $(n=230)$ & $\begin{array}{c}\text { Grindr user } \\
(n=89)\end{array}$ & $\begin{array}{c}\text { Non-Grindr app user } \\
(n=138)\end{array}$ & $p$ \\
\hline & \multicolumn{4}{|c|}{$\mathrm{n}(\%)$ or mean (SD), as appropriate } & \\
\hline Age & $41.1 \pm 12.7(18-74)$ & $42.5 \pm 13.0(18-74)$ & $39.9 \pm 13.4(18-62)$ & $39.4 \pm 11.6(18-65)$ & $<0.05$ \\
\hline $\begin{array}{l}\text { Gender } \\
\quad \text { Male } \\
\text { Transgender }\end{array}$ & $\begin{array}{c}451(98.7) \\
6(1.3)\end{array}$ & $\begin{array}{c}225(97.8) \\
5(2.2)\end{array}$ & $\begin{array}{c}89(100.0) \\
0(0.0)\end{array}$ & $\begin{array}{c}137(99.8) \\
1(0.7)\end{array}$ & 0.34 \\
\hline $\begin{array}{l}\text { Race/ethnicity } \\
\text { White } \\
\text { Non White }\end{array}$ & $\begin{array}{l}376(82.3) \\
81(17.7)\end{array}$ & $\begin{array}{l}195(84.8) \\
35(15.2)\end{array}$ & $\begin{array}{l}76(85.4) \\
13(14.6)\end{array}$ & $\begin{array}{l}105(76.1) \\
33(23.9)\end{array}$ & 0.07 \\
\hline $\begin{array}{l}\text { Sexual orientation } \\
\text { Gay/homosexual } \\
\text { Bisexual } \\
\text { Heterosexual/other }\end{array}$ & $\begin{array}{l}209(45.7) \\
186(40.7) \\
62(13.6)\end{array}$ & $\begin{array}{l}80(34.8) \\
108(47.0) \\
42(18.3)\end{array}$ & $\begin{array}{l}58(65.2) \\
25(28.1) \\
6(6.7)\end{array}$ & $\begin{array}{l}71(51.4) \\
53(38.4) \\
14(10.1)\end{array}$ & $<0.001$ \\
\hline Outness & $2.4 \pm 1.2(1-4)$ & $2.1 \pm 1.2(1-4)$ & $2.8 \pm 1.2(1-4)$ & $2.7 \pm 1.2(1-4)$ & $<0.001$ \\
\hline Tested for HIV ${ }^{1}$ & $358(78.3)$ & $177(77.0)$ & $71(79.8)$ & $110(79.7)$ & 0.78 \\
\hline Tested for HIV ${ }^{2}$ & $206(45.1)$ & $97(55.4)$ & $44(49.4)$ & $65(59.1)$ & 0.61 \\
\hline Positive HIV status & $42(9.2)$ & $4(1.7)$ & $19(21.3)$ & $19(13.8)$ & $<0.001$ \\
\hline $\begin{array}{l}\text { Location of last HIV test } \\
\text { Traditional } \\
\text { Non-traditional }\end{array}$ & $\begin{array}{l}181(39.6) \\
276(60.4)\end{array}$ & $\begin{array}{c}82(35.7) \\
148(64.3)\end{array}$ & $\begin{array}{l}49(55.1) \\
40(44.9)\end{array}$ & $\begin{array}{l}50(36.2) \\
88(63.8)\end{array}$ & 0.004 \\
\hline $\begin{array}{l}\text { HIV testing self-efficacy } \\
\text { Less than very sure } \\
\text { Very sure }\end{array}$ & $\begin{array}{c}85(18.6) \\
372(81.4)\end{array}$ & $\begin{array}{l}67(29.1) \\
163(70.9)\end{array}$ & $\begin{array}{c}7(7.9) \\
82(92.1)\end{array}$ & $\begin{array}{c}11(8.0) \\
127(92.0)\end{array}$ & $<0.001$ \\
\hline Sex with a female partner ${ }^{2}$ & $214(46.8)$ & $134(58.3)$ & $25(28.1)$ & $55(39.9)$ & $<0.001$ \\
\hline Inconsistent condom use ${ }^{2}$ & $328(71.8)$ & $177(77.0)$ & $54(60.7)$ & $97(70.3)$ & 0.01 \\
\hline Days drunk in a typical week & $0.4 \pm 1.1(0-6)$ & $0.5 \pm 1.2(0-6)$ & $0.3 \pm 0.6(0-4)$ & $0.4 \pm 0.9(0-4)$ & 0.13 \\
\hline Tobacco (cigarette or cigar) use ${ }^{2}$ & $177(38.7)$ & $90(39.1)$ & $48(53.9)$ & $39(28.3)$ & 0.001 \\
\hline Marijuana use $^{2}$ & $0(0.0)$ & $0(0.0)$ & $0(0.0)$ & $0(0.0)$ & -- \\
\hline Cocaine use ${ }^{2}$ & $12(2.6)$ & $9(3.9)$ & $2(2.2)$ & $1(0.7)$ & 0.21 \\
\hline Crack use $^{2}$ & $0(0.0)$ & $0(0.0)$ & $0(0.0)$ & $0(0.0)$ & -- \\
\hline Poppers/Rush use ${ }^{2}$ & $75(16.4)$ & $13(5.7)$ & $26(29.2)$ & $36(26.1)$ & $<0.001$ \\
\hline Crystal Meth use ${ }^{2}$ & $0(0.0)$ & $0(0.0)$ & $0(0.0)$ & $0(0.0)$ & -- \\
\hline Ecstasy use $^{2}$ & $11(2.4)$ & $6(2.6)$ & $0(0.0)$ & $5(3.6)$ & 0.22 \\
\hline 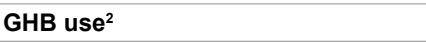 & $0(0.0)$ & $0(0.0)$ & $0(0.0)$ & $0(0.0)$ & -- \\
\hline Erectile dysfunction medication ${ }^{2}$ & $87(19.0)$ & $43(18.7)$ & $18(20.2)$ & $26(18.8)$ & 0.96 \\
\hline Other illicit substances ${ }^{2}$ & $3(0.7)$ & $1(0.4)$ & $2(2.2)$ & $0(0.0)$ & 0.13 \\
\hline Recreational prescription drug use ${ }^{1}$ & $44(9.6)$ & $21(9.4)$ & $19(21.3)$ & $4(2.9)$ & $<0.001$ \\
\hline
\end{tabular}

${ }^{1}$ lifetime. ${ }^{2}$ within the past 12 months.

Table 1: Sample characteristics and differences by app use (bivariable analysis) 
Citation: Sun CJ, Sutfin EL, Bachmann LH, Stowers J, Rhodes SD (2018) Comparing Men who have Sex with Men and Transgender Women who Use Grindr, Other Similar Social and Sexual Networking Apps, or no Social and Sexual Networking Apps: Implications for Recruitment and Health Promotion. J AIDS Clin Res 9: 757. doi: 10.4172/2155-6113.1000757

Page 4 of 6

\section{Description of app use}

Half $(50.3 \%, \mathrm{n}=230)$ of the participants were non-app users; $19.5 \%$ $(\mathrm{n}=89)$ were Grindr users; $30.2 \%(\mathrm{n}=138)$ were non-Grindr app users. Among all the app users (both Grindr users and non-Grindr app users), the most frequently reported apps were A4A/Radar, Grindr, Jack'd, and Scruff; $80.1 \%$ of all app users reported using at least one of these apps. The least frequently reported apps were BoyAhoy and Hornet. Hornet was reported by only two individuals, both of whom used other apps as well. BoyAhoy was reported by 5 individuals as the only app they used. No participants reported using Mister.

Among the Grindr-users, $77.5 \%$ used Grindr only. Of the other apps used by Grindr users, A4A/Radar was the most frequently reported $(n=17)$; all of the other apps were only reported by 1 or 2 other participants.

Among the non-Grindr app users, 79.0\% reported using only 1 app in the past 30 days. Non-Grindr app users most frequently reported using A4A/Radar ( $n=46)$, followed by Scruff $(n=36)$, and Jack'd $(n=25)$. BoyAhoy and Hornet were the least frequently reported apps $(\mathrm{n}=5$ and $\mathrm{n}=2$, respectively).

\section{Comparison of HIV risk and substance use by app use}

From the bivariable analyses, there were significant differences in the sociodemographic characteristics by app use, including age, race/ ethnicity, sexual orientation, and outness (Table 1). On average, nonapp users were 2.6 years older than Grindr users and 3.1 years older than non-Grindr app users. Fewer non-Grindr app users were White compared to non-app users and Grindr users $(76.1 \%$ versus $84.9 \%$ and $85.4 \%$, respectively). Nearly twice as many Grindr users identified as gay/homosexual compared to non-app users. Grindr users reported the highest outness score and non-app users had the lowest outness score.

After adjusting for the sociodemographic characteristics significantly associated with app use, there were significant differences in HIV risk and substance use between the groups, as presented in Table 2. Non-app users compared to Grindr users were less likely to report a positive HIV status as well as popper and recreational prescription drug use, though they were more likely to report inconsistent condom use and lower HIV testing self-efficacy. Non-Grindr app users compared to Grindr users were less likely to report tobacco (cigarette or cigar) and recreational prescription drug use, and more likely to report inconsistent condom use.

\section{Discussion}

We found significant differences in sociodemographic characteristics when we compared a sample of MSM and transgender women who reported using Grindr, not using Grindr but using similar apps, or not using any GPS-based social and sexual networking apps. MSM and transgender women who reported not using any GPS-based social and sexual networking apps were more likely to be older and identify as bisexual or heterosexual compared to those who reported using apps. There were additional differences in HIV-related characteristics and behaviors after controlling for sociodemographic characteristics, including HIV status, HIV testing self-efficacy, and substance use.

This paper is the first of its kind to report on findings that compare MSM and transgender women who report using Grindr to MSM and transgender women who report not using Grindr but using other similar apps. As such, we are unable to compare our findings to other published papers. As important differences were identified between these two groups, further research could seek to replicate these results and continue to examine how MSM and transgender women who use Grindr and MSM and transgender women who do not use Grindr but use similar apps are different or similar from one another. Furthermore, our sample included very few transgender women; however, their use of GPS-based apps may be increasing and research should better explore their patterns of use. The differences in sociodemographic characteristics that were identified by comparing MSM and transgender women who did not report using apps to MSM and transgender women who reported using apps are similar to other findings $[8,33]$.

These findings have important recruitment implications. GPS-based social and sexual networking apps may offer a valuable recruitment tool for future HIV research seeking to recruit populations at increased risk for HIV or those living with HIV for therapeutic trials. Additionally, it appears that if we were interested in recruiting MSM and transgender women with certain sociodemographic characteristics, such as MSM and transgender women of color, older MSM and transgender women, or less out MSM and transgender women, we should consider using other apps in addition to Grindr or non-app venues. Because of the differences identified across users of different apps, these findings suggest that if researchers recruited participants from just Grindr, they could end up with a sample quite different than if they had recruited MSM and transgender women from other apps.

In terms of intervention implications, researchers and practitioners could apply these findings to reach different target groups and implement interventions that encourage increasing HIV testing and condom use or decreasing substance use. For example, MSM and transgender women who do not report using GPS-based social and sexual networking apps were more likely to report low HIV testing self-efficacy, which is an important predictor of actual HIV testing, a critical aspect of HIV prevention. It may be necessary to connect with these populations through other venues in order to provide support

\begin{tabular}{|c|c|c|c|c|}
\hline & $\begin{array}{l}\text { Non-app user compared to } \\
\text { Grindr user }{ }^{1}\end{array}$ & $p$ & $\begin{array}{c}\text { Non-Grindr app user compared to } \\
\text { Grindr user }{ }^{1}\end{array}$ & $p$ \\
\hline & $\operatorname{AOR}(95 \% \mathrm{Cl})$ & & $\operatorname{AOR}(95 \% \mathrm{Cl})$ & \\
\hline Positive HIV status & $0.05(0.01,0.16)$ & $<0.001$ & $0.45(0.20,1.01)$ & 0.053 \\
\hline Last HIV testing at a traditional venue (compared to non-traditional) & $0.78(0.38,1.62)$ & 0.51 & $0.79(0.38,1.64)$ & 0.53 \\
\hline Less than very sure HIV testing self-efficacy (compared to very sure) & $4.56(1.85,11.24)$ & 0.001 & $1.04(0.36,3.02)$ & 0.94 \\
\hline Sex with a female partner ${ }^{2}$ & $1.75(0.81,3.79)$ & 0.15 & $1.80(0.80,4.05)$ & 0.16 \\
\hline Inconsistent condom use ${ }^{2}$ & $2.42(1.35,4.36)$ & 0.003 & $2.49(1.34,4.62)$ & 0.004 \\
\hline Tobacco (cigarette or cigar) use ${ }^{2}$ & $0.83(0.47,1.47)$ & 0.51 & $0.34(0.18,0.64)$ & 0.001 \\
\hline Popper use ${ }^{2}$ & $0.14(0.07,0.32)$ & $<0.001$ & $1.15(0.59,2.22)$ & 0.69 \\
\hline Recreational prescription drug use $^{3}$ & $0.36(0.17,0.77)$ & 0.009 & $0.11(0.03,0.34)$ & $<0.001$ \\
\hline
\end{tabular}

${ }^{1}$ Grindr user is the referent group. AOR and $95 \% \mathrm{Cl}$ adjusted for age, geographic region, race/ethnicity, sexual orientation, and outness. ${ }^{2}$ within the past 12 months. ${ }^{3}$ lifetime. Table 2: Comparison of HIV risk and substance use by app use: Multivariable logistic regression analysis ( $n=457)$. 
Citation: Sun CJ, Sutfin EL, Bachmann LH, Stowers J, Rhodes SD (2018) Comparing Men who have Sex with Men and Transgender Women who Use Grindr, Other Similar Social and Sexual Networking Apps, or no Social and Sexual Networking Apps: Implications for Recruitment and Health Promotion. J AIDS Clin Res 9: 757. doi: 10.4172/2155-6113.1000757

Page 5 of 6

to increase HIV testing self-efficacy. Research has consistently shown that MSM and transgender women have higher rates of smoking than their heterosexual counterparts $[34,35]$. Developing and evaluating the efficacy of HIV and substance use interventions delivered via apps could be an important next step to reduce health disparities, as researchers have already begun to demonstrate the acceptability and feasibility of promoting sexual health and HIV prevention on GPS-based social and sexual networking apps.

\section{Strengths}

This is the first paper to systematically describe and compare the sociodemographic characteristics, HIV risk, and substance use among MSM and transgender women who report using a variety of GPSbased social and sexual networking apps. Additionally, this research was conducted in North Carolina, which is a fairly rural state with a unique social context, and contributes to increasing our knowledge and understanding of MSM and transgender women outside of large, urban areas, such as Los Angeles, New York City, and Atlanta where most of the app-based research has been conducted [4-7,9,11,13,15,17]. Furthermore, given the rising and disproportionate HIV rates in the South and that the $14^{\text {th }}$ and $22^{\text {nd }}$ highest rates of HIV occurred in two metropolitan statistical areas in North Carolina, [1,36] conducting research to explore HIV risk in this area is greatly needed and should be a high priority in order to address the epidemiological changes in HIV in the United States.

\section{Limitations}

There are several limitations of this analysis that impact the interpretation of results. The results of this analysis are based on cross-sectional data, and no causal relationships can be assumed. The substance use measures lack specificity; participants were asked to report their substance use in the past 12 months and we did not measure frequency and intensity of use or routes of administration. Future research should seek to better understand these important features. It would have been insightful to recruit men and transgender women from social and sexual networking apps, and then conduct analyses to compare the various samples; however, by recruiting from these platforms, we were able to make comparisons between app users and non-app users.

\section{Conclusion}

These findings suggest that researchers need to carefully consider differences in samples that may exist based on the social media platform from where participants are recruited. We need to expand our understanding of apps as a new venue for recruitment, and there are important implications in the differences of the individuals we reach when recruiting from these new apps. Given the changing context and technologies, researchers and interventionists must understand and utilize venues that span beyond physical space to include new and virtual spaces that are popular amongst MSM and transgender women to socialize and meet, and at the same time continue to reach and meet the needs of all MSM and transgender women, including incorporating non-app-based strategies.

\section{Acknowledgement}

We sincerely thank the study participants for their time and openness.

\section{Contributors}

CJS, ES, and SDR shared in the conception and design of the project. Data acquisition, analysis, and interpretation were carried out by JS, CJS, SDR, and LB. CJS and SDR drafted the manuscript. All authors participated in critical revision and final approval of the manuscript.

\section{Financial Support}

This material is based upon support from the National Institutes of Health (R01MH092932) and the Agency for Healthcare Research and Quality (K12HS02298). The content is solely the responsibility of the authors and does not necessarily represent the official views of the National Institutes of Health and Agency for Healthcare Research and Quality.

\section{References}

1. Centers for Disease Control and Prevention (2015) HIV surveillance report CDC 2013: 25

2. Centers for Disease Control and Prevention (2012) Estimated HIV incidence in the United States, 2007-2010. HIV SurveillSuppl Rep 17.

3. Johnson AS, Beer L, Sionean C, Hu X, Furlow-Parmley C, et al. (2013) HIV infection — United States, 2008 and 2010. MMWR 62: 112-119.

4. Rice E, Holloway I, Winetrobe $H$, Rhoades $H$, Barman-Adhikari $A$, et al (2012) Sex risk among young men who have sex with men who use Grindr, a smartphone geosocial networking application. J AIDS Clin Res 1: 005.

5. Phillips G 2nd, Magnus M, Kuo I, Rawls A, Peterson J, et al. (2014) Use of geosocial networking (GSN) mobile phone applications to find men for sex by men who have sex with men (MSM) in Washington, DC. AIDS Behav 18: 1630 1637.

6. Beymer MR, Weiss RE, Bolan RK, Rudy ET, Bourque LB, et al. (2014) Sex on demand: Geosocial networking phone apps and risk of sexually transmitted infections among a cross-sectional sample of men who have sex with men in Los Angeles County. Sex Transm Infect 90: 567-572.

7. Goedel WC, Duncan DT (2015) Geosocial-networking app usage patterns of gay, bisexual, and other men who have sex with men: Survey among users of Grindr, a mobile dating app. JMIR Public Heal Surveill 1: e4.

8. Lehmiller JJ, loerger M (2014) Social networking smartphone applications and sexual health outcomes among men who have sex with men. PLoS One 9: e86603.

9. Holloway IW, Pulsipher CA, Gibbs J, Barman-Adhikari A, Rice E (2015) Network influences on the sexual risk behaviors of gay, bisexual and other men who have sex with men using geosocial networking applications. AIDS Behav 19: $112-122$.

10. Brooks RA, Landovitz RJ, Kaplan RL, Lieber K, Lee SJ, et al. (2012) Sexua risk behaviors and acceptability of HIV pre-exposure prophylaxis among HIV-negative gay and bisexual men in serodiscordant relationships: A mixed methods study. AIDS Patient Care STDs 26: 87-94.

11. Rendina HJ, Jimenez RH, Grov C, Ventuneac A, Parsons JT (2014) Patterns of lifetime and recent HIV testing among men who have sex with men in New York City who use Grindr. AIDS Behav 18: 41-49.

12. Su JY, Holt J, Payne R, Gates K, Ewing A, et al. (2015) Effectiveness of using Grindr to increase syphilis testing among men who have sex with men in Darwin, Australia. Aust N Z J Public Health 39: 293-294.

13. Rosengren AL, Huang E, Daniels J, Young SD, Marlin RW, et al. (2016) Feasibility of using GrindrTM to distribute HIV self-test kits to men who have sex with men in Los Angeles, California. Sex Health.

14. Lampkin D, Crawley A, Lopez TP, Mejia CM, Yuen W, et al. (2016) Reaching suburban men who have sex with men for STD and HIV services through online social networking outreach: A public health approach. J Acquir Immune Defic Syndr 72: 73-78.

15. Landovitz RJ, Tseng CH, Weissman M, Haymer M, Mendenhall B, et al. (2013) Epidemiology, sexual risk behavior, and HIV prevention practices of men who have sex with men using GRINDR in Los Angeles, California. J Urban Heal 90: 729-739.

16. Burrell ER, Pines HA, Robbie E, Coleman L, Murphy R, et al. (2012) Use of the location-based social networking application GRINDR as a recruitment tool in rectal microbicide development research. AIDS Behav 16: 1816-1820.

17. Winetrobe H, Rice E, Bauermeister J, Petering R, Holloway IW (2014) Associations of unprotected anal intercourse with Grindr-met partners among Grindr-using young men who have sex with men in Los Angeles. AIDS Care 26: 1303-1308.

18. Blackwell C, Birnholtz J, Abbott C (2015) Seeing and being seen: Co-situation and impression formation using Grindr, a location-aware gay dating app. New Media Soc 17: 1117-1136. 
Citation: Sun CJ, Sutfin EL, Bachmann LH, Stowers J, Rhodes SD (2018) Comparing Men who have Sex with Men and Transgender Women who Use Grindr, Other Similar Social and Sexual Networking Apps, or no Social and Sexual Networking Apps: Implications for Recruitment and Health Promotion. J AIDS Clin Res 9: 757. doi: 10.4172/2155-6113.1000757

Page 6 of 6

19. Brubaker JR, Ananny M, Crawford K (2016) Departing glances: A sociotechnical account of "leaving" Grindr. New Media Soc 18: 373-390.

20. Grov C, Breslow AS, Newcomb ME, Rosenberger JG, Bauermeister JA (2014) Gay and bisexual men's use of the Internet: Research from the 1990s through 2013. J Sex Res 51: 390-409.

21. Ramo DE, Hall SM, Prochaska JJ (2010) Reaching young adult smokers through the Internet: Comparison of three recruitment mechanisms. Nicotine \& Tobacco Research 12: 768-775.

22. Rhodes SD (2014) Innovations in HIV Prevention Research and Practice through Community Engagement. Springer, New York, NY.

23. Sun CJ, Stowers J, Miller C, Bachmann LH, Rhodes SD (2014) Acceptability and feasibility of using established geosocial and sexual networking mobile applications to promote HIV and STD testing among men who have sex with men. AIDS and Behavior 9: 543-552.

24. Rhodes SD, Hergenrather KC, Yee LJ, Ramsey B (2008) Comparing MSM in the southeastern United States who participated in an HIV prevention chat room-based outreach intervention and those who did not: how different are the baseline HIV-risk profiles? Health Educ Res 23: 180-190.

25. Rhodes SD, Hergenrather KC, Yee LJ, Knipper E, Wilkin AM, et al. (2007) Characteristics of a sample of men who have sex with men, recruited from gay bars and Internet chat rooms, who report methamphetamine use. AIDS Patien Care STDS 21: 575-583.

26. Rhodes SD, Bowie DA, Hergenrather KC (2003) Collecting behavioural data using the world wide web: considerations for researchers. J Epidemiol Community Health 57 : 68-73.

27. Rhodes SD, Hergenrather KC, Duncan J, Vissman AT, Miller C, et al. (2010) A pilot intervention utilizing Internet chat rooms to prevent HIV risk behaviors among men who have sex with men. Public Health Rep 125 Suppl 1: 29-37.
28. Rhodes SD, Vissman AT, Stowers J, Miller C, McCoy TP, et al. (2011) A CBPR partnership increases HIV testing among men who have sex with men (MSM) Outcome findings from a pilot test of the CyBER/testing internet intervention. Health Educ Behav 38: 311-320.

29. Rhodes SD, DiClemente RJ, Cecil H, Hergenrather KC, Yee LJ (2002) Risk among men who have sex with men in the United States: A comparison of an Internet sample and a conventional outreach sample. AIDS Educ Prev 14: $41-50$.

30. O'Brien MC, McCoy TP, Champion H, Mitra A, Robbins A, et al. (2006) Single question about drunkenness to detect college students at risk for injury. Acad Emerg Med 13: 629-636.

31. Rhodes SD, McCoy TP, Hergenrather KC, Vissman AT, Wolfson M, et al. (2012) Prevalence estimates of health risk behaviors of immigrant Latino men who have sex with men. J Rural Heal 28: 73-83.

32. Hosmer DW, Lemeshow S (1989) Applied Logistic Regression. Wiley, New York.

33. Bien CH, Best JM, Muessig KE, Wei C, Han L, et al. (2015) Gay apps for seeking sex partners in China: Implications for MSM sexual health. AIDS Behav 19: 941-946.

34. Lee JGL, Blosnich JR, Melvin CL (2012) Up in smoke: Vanishing evidence of tobacco disparities in the Institute of Medicine's report on sexual and gender minority health. Am J Public Health 102: 2041-2043.

35. Lee JG, Griffin GK, Melvin CL (2009) Tobacco use among sexual minorities in the USA, 1987 to May 2007: A systematic review. Tob Control 18: 275-282.

36. Reif SS, Whetten K, Wilson ER, McAllaster C, Pence BW, et al. (2014) HIV/ AIDS in the Southern USA: A disproportionate epidemic. AIDS Care 26: 351 359 